Derleme Makalesi - Review Article

\title{
Bazı Ultranormlu Uzaylar ve İzomorfikliği
}

\author{
İbrahim ŞANLIBABA ${ }^{1 *}$ \\ Geliş / Received: 04/03/2020 \\ Revize / Revised: 28/05/2020 \\ Kabul / Accepted: 01/06/2020 \\ $\overline{\mathbf{O Z Z}}$ \\ Bu çalışmada metrik uzaylar, ultrametrik uzayları, normlu uzaylar ve ultranormlu uzaylar tanıtılıp aralarındaki \\ ilişsiler ve örnekleri gösterildi. Ultranormlu uzaylarda izometri ve ultra izometri tanımları yapıldı. Sonra bazı \\ ultranormlu uzaylara örnekler verilip ultra Banach uzay olduğu ve izomorfik olduğu gösterildi.
}

Anahtar Kelimeler- Ultrametrik, Ultranorm, Krull Özelliği, Ultra İzometri, Ultra Banach Uzayları.

1*Sorumlu yazar iletişim: ibrahimsanlibaba@gmail.com (https://orcid.org/0000-0001-8801-464X)

Matematik Bölümü, Nevşehir Hacı Bektaş Veli Üniversitesi, Nevșehir. 


\title{
The Some Ultranorm Spaces and Isomorphicity
}

\begin{abstract}
In this study metric spaces, ultrametric spaces, normed spaces and ultranormed spaces are introduced and their relations between each other and examples are shown. Isometry and ultra isometry are defined in ultranormed spaces. Then some example of ultra-normed spaces are given and it is shown to be ultra Banach space and is isomorphic.
\end{abstract}

Keywords- Ultrametric, Ultranorm, Krull Sharpening, Ultra Isometry, Ultra Banach Spaces. 


\section{GİRIŞ}

$\mathrm{Bu}$ çalışmada ultrametrik uzay kavramının belirteci olan üçüncüsü aksiyomun $\mathrm{d}(\mathrm{x}, \mathrm{y}) \leq$ $\operatorname{mak}\{\mathrm{d}(\mathrm{x}, \mathrm{z}), \mathrm{d}(\mathrm{z}, \mathrm{y})\}$ alınmasıyla oluşturulmuş metriğe ultrametrik ismi verilmiş ayrıca ilginç, dikkat çekici neticeler çıkarılmıştır. Sonra ultrametrik ile metrik uzayları, ultranormlu ile normlu uzayları ve ilişkileri gösterilmiştir. Bu aksiyom ile ultrametrik uzayları, ultranormu, ultrametrik Banach uzaylarını ve ultrametrik Banach uzaylarının değişik özellikleri incelenmiştir. Ayrıca Diagana, Krull, Ludkovsky ve Şanlıbaba [1,2,3,4] ultrametrik uzayların yapılarını, bu uzaylara örnekleri ve kapsama durumlarını göstermişlerdir. Sonuç olarak bazı farklı yapılar ve uzaylar ortaya çıkarılmış, tanımlamaları ve örnekleri açıklanmıştır.

\section{TEMEL TANIM VE TEOREMLER}

Tanım 2.1. $\mathrm{X}$ bir küme olmak üzere ve $\mathrm{X}$ üzerinde tanımlı $\mathrm{d}: \mathrm{X} \mathrm{XX} \rightarrow \mathbb{R}$ bir $d$ fonksiyonu verilmek üzere $\forall \mathrm{x}, \mathrm{y}, \mathrm{z} \in \mathrm{X}$ için $d$ fonksiyonu M1- M3 aksiyomlarını sağlıyorsa $d$ fonksiyonuna metrik, (X, d) ikilisine de metrik uzay denir.

$$
\begin{aligned}
& \text { (M1) } d(x, y)=0 \Leftrightarrow x=y \\
& \text { (M2) } d(x, y)=d(y, x) \text { (Simetri) } \\
& \text { (M3) } d(x, y) \leq d(x, z)+d(z, y) \text { (Üçgen eşitsizliği) }
\end{aligned}
$$

Pozitif olan d $(\mathrm{x}, \mathrm{y})$ ile gösterilen negatif olmayan reel sayısına x ile y arasındaki uzaklık denir [5].

Ultrametrik uzaylar aslında metrik uzayların daha özel hali olup aşağıda tanımı gösterilecek ve karşılaştırmalar yapılacak, ultrametriklere örnekler verilecektir.

Tanım 2.2. $\mathrm{X}$ bir küme ve $\forall \mathrm{x}, \mathrm{y}, \mathrm{z} \in \mathrm{X}$ için $\mathrm{d}_{\mathrm{u}}: \mathrm{Xx} X \rightarrow \mathbb{R}$ fonksiyonu verilsin. $\mathrm{d}_{\mathrm{u}}$ fonksiyonu;

$$
\begin{aligned}
& \text { (UM1) } d_{u}(x, y)=0 \Leftrightarrow x=y \\
& (U M 2) d_{u}(x, y)=d_{u}(y, x) \text { (Simetri) } \\
& \text { (UM3) } d_{u}(x, y) \leq \operatorname{mak}\left\{d_{u}(x, z), d_{u}(z, y)\right\} \text { (Güçlü üçgen eşitsizliği) }
\end{aligned}
$$

koşullarını sağlıyorsa $d_{u}{ }^{\prime}$ ya ultrametrik, $\left(X, d_{u}\right)$ veya ultrametrik uzay, (UM1), (UM2) ve (UM3) şartlarını da ultrametrik uzay aksiyomları denilir.

Metrik ve ultrametrik koşulları incelenirse (UM1) aksiyomu (M1) aksiyomu ile, (UM2) aksiyomu (M2) aksiyomu ile aynıdır. Ultrametrik üçüncü aksiyomda $d(x, y) \leq \operatorname{mak}\{d(x, z), d(z, y)\}$ eşitsizliğinde maksimum değer $d(x, z)$ olursa $d(x, y) \leq d(x, z)+d(z, y)$ eşitsizliğin sağlandığı açıktır. Diğer durumda maksimum değer $d(z, y)$ olarak seçilirse aynı şekilde $d(x, y) \leq d(x, z)+d(z, y)$ eşitsizliği yine korunur. Sonuç olarak (UM3) eşitsizliğinden (M3) eşitsizliği elde edilir. Diğer taraftan her zaman (M3) den (UM3) elde edilemeyeceğinden her metrik uzay bir ultrametrik uzay değildir. Dolayısıyla her ultrametrik uzay bilinen anlamda metrik uzay olur fakat tersi olmayabilir.

$\mathrm{d}: \mathbb{R} \mathrm{x} \mathbb{R} \rightarrow \mathbb{R}$ ve $\mathrm{d}(\mathrm{x}, \mathrm{y})=|\mathrm{x}-\mathrm{y}|$ olarak tanımlanan $\mathbb{R}$ deki doğal metriği düşünecek olunursa d'nin M1-M3 koşullarını sağladığı açık olarak görülür. Gerçekten de mutlak değer metriği ile $\mathbb{R}$ gözönüne alındığında $\mathrm{x}=-2, \mathrm{y}=-5, \mathrm{z}=-3$ alarak $\mathrm{d}(\mathrm{x}, \mathrm{y})=3, \mathrm{~d}(\mathrm{x}, \mathrm{z})=1, \mathrm{~d}(\mathrm{z}, \mathrm{y})=2$ olup güçlü üçgen eşitsizliğinin sağlanmadığ görülebilir. Dolayısıyla $d, \mathbb{R}$ de bir metrik olduğu halde (UM3) koşulunu sağlamadığından ultrametrik olmaz. Sonuçta $(\mathbb{R}, d)$ ultrametrik uzay değildir.

Şimdi takip eden teorem verilebilir:

Teorem 2.1. Bütün ultrametrik uzaylar aslında bir metrik uzaydır fakat her metrik uzay bir ultrametrik uzay olmayabilir. 
Örnek 1. $X \neq \varnothing$ bir küme verilmiş olmakla birlikte, $X \times X \rightarrow \mathbb{R}$

$d_{u}(x, y)=\left\{\begin{array}{l}0, x=y \\ 1, x \neq y\end{array}\right.$ şeklinde tanımlanan

$\mathrm{d}_{\mathrm{u}}$ fonksiyonu $\mathrm{X}$ de bir ultrametriktir [6], [7].

Tanım 2.3. $\mathrm{X}$ kümesi verilsin ve $\mathrm{X}$ in birimi $\theta$ ile gösterilmiş olan $\forall \mathrm{x} \in \mathrm{X}$ için $0+\mathrm{x}=0$ olmak üzere $\mathrm{X}$ kümesinde toplama ve skalarla çarpma işlemleri tanımlansın.

$\forall \mathrm{x}, \mathrm{y} \in \mathrm{X}$ ve $\forall \alpha \in \mathbb{C}$ olacak şekilde $\mathrm{g}: \mathrm{X} \rightarrow \mathbb{R}$ fonksiyonu

(UN1) $\mathrm{g}(\mathrm{x})=0 \Leftrightarrow \mathrm{x}=\theta$

(UN2) $g(\alpha x)=|\alpha| g(x)$

(UN3) $\mathrm{g}(\mathrm{x}+\mathrm{y}) \leq \operatorname{mak}\{\mathrm{g}(\mathrm{x}), \mathrm{g}(\mathrm{y})\}$

koşullarını sağlarsa $\mathrm{g}$ fonksiyonuna $\mathrm{X}$ de ultranorm denir. Norm ve ultranorm aksiyomları karşılaştırıldığında (UN3) ve (N3) aksiyomları birbirinden ayrı olduğu gözlemlenir.

$\mathrm{g}(\mathrm{x}+\mathrm{y}) \leq \operatorname{mak}\{\mathrm{g}(\mathrm{x}), \mathrm{g}(\mathrm{y})\}$ eşitsizliği $\mathrm{g}(\mathrm{x}+\mathrm{y}) \leq \mathrm{g}(\mathrm{x})+\mathrm{g}(\mathrm{y})$ eşitsizliğini gerektirirken $\mathrm{g}(\mathrm{x}+\mathrm{y}) \leq$ $\mathrm{g}(\mathrm{x})+\mathrm{g}(\mathrm{y})$ eşitsizliği $\mathrm{g}(\mathrm{x}+\mathrm{y}) \leq \operatorname{mak}\{\mathrm{g}(\mathrm{x}), \mathrm{g}(\mathrm{y})\}$ eşitsizliğini gerektirmez. Böylece her ultranorm bir normdur fakat tersi doğru değildir.

Her normun bir metrik olduğu bilinen bir önermedir. Benzer şekilde ultrametrik ve ultranorm içinde durum aynıdır. Yani "Her ultranorm bir ultrametriktir" [6].

Sonuç olarak normlu uzayların birinci ve ikinci şartı ile ultranormlu uzayların birinci ve ikinci şartı aynıdır. Yine normlu uzayların üçüncü şartı olan üçgen eşitsizliği koşulunun $\|x+y\| \leq \operatorname{mak}\{\|x\|,\|y\|\}$ eşitsizliği var iken sağlandığı ama $\|x+y\| \leq\|x\|+\|y\|$ eşitsizliğinin var olmasının $\|x+y\| \leq \operatorname{mak}\{\|x\|,\|y\|\}$ eşitsizliğinin mevcut olmasını gerektirmeyeceği görülür. Dolayısıyla "Her ultranormlu uzay normlu uzaydır, tersine her normlu uzay ultranormlu uzay olmayabilir” önermesi verilebilir.

Buraya kadar anlatılanları ve ilişkilerini aşağıdaki diyagram özetler:

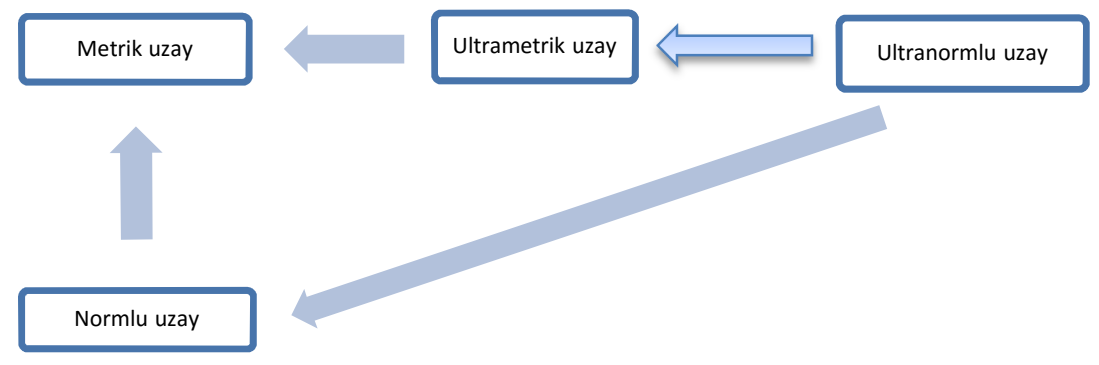

Diyagram 1. Metrik ve Ultrametrik Uzay, Normlu ve Ultranormlu Uzay İlişkileri arasındaki bağıntıyı gösteren diyagram

Tanım 2.4. Bir X ultranormlu (Arşimedyan olmayan) lineer uzayı tam ise X uzayına Ultra Banach uzayı ya da Arşimedyan olmayan Banach uzayı denir.

Tanım 2.5. X bir normlu uzay ve $\forall x, y \in X$ olsun. $\|x\| \neq\|y\|$ olduğunda $\|x+y\| \leq \operatorname{mak}(\|x\|,\|y\|)$ eşitsizliği sağlanıyorsa \|. \| ye Krull özelliğine sahiptir denir [2]. 


\section{BAZI ULTRANORMLU UZAYLAR VE IZOMORFIKLLIKLERİ}

Tanım 3.1. $\mathrm{T}: \mathrm{X} \rightarrow \mathrm{Y}$ dönüşümü verilsin. $\mathrm{X}$ ve $\mathrm{Y}, \mathrm{K}$ cismi üzerinde ultranormlu uzaylar olsun. $\forall \mathrm{x} \in \mathrm{X}$ olmak üzere;

$$
\|\mathrm{T}(\mathrm{x})\|=\|\mathrm{x}\|
$$

eşitliği varsa kısacası ultranormu koruyorsa, T nin bir ultra izometri olduğu söylenir.

K cismi üzerinde $X$ ve $Y$ ultranormlu uzaylar olarak verilsin. $T: X \rightarrow Y$ dönüşümü lineer, birebir, örten ve izometri mevcutsa $X$ ve $Y$ uzaylarına ultra izometrik olarak ultra izomorfiktir denir ayrica bu durum $X_{u} \cong Y_{u}$ ile ifade edilir.

$\mathrm{K}$ bir cisim olmak üzere $\forall \mathrm{x}, \mathrm{y} \in \mathrm{K}$ için $||:. \mathrm{K} \rightarrow \mathbb{R}$ fonksiyonu (d3)' ultrametrik 3.şartı olan $|\mathrm{x}+\mathrm{y}| \leq \operatorname{mak}\{|\mathrm{x}|,|\mathrm{y}|\}$ eşitsizliğini sağlasın. $\forall \mathrm{k} \in \mathbb{N}$ için $\left(\rho_{\mathrm{k}}\right) ; \mathbb{R}^{+}-0$ biçiminde $\left(\rho_{\mathrm{k}}\right)$ dizisi sınırlı bir dizi ve I da bir indis kümesi olacak şekilde, $\mathrm{K}$ de;

$$
l_{\infty}(\mathrm{I}, \mathrm{K}, \rho)=\left\{\mathrm{x}=\left(\mathrm{x}_{\mathrm{k}}\right) \in \mathrm{K}: \sup _{\mathrm{k} \in \mathbb{N}}\left|\mathrm{x}_{\mathrm{k}}\right| \rho_{\mathrm{k}}<\infty\right\}
$$

kümesini tanımlayalım.

$$
\begin{aligned}
\|\cdot\|: l_{\infty}(\mathrm{I}, \mathrm{K}, \rho) & \rightarrow \mathbb{R} \\
\mathrm{x} & \rightarrow\|\mathrm{x}\|=\sup _{\mathrm{k}}\left|\mathrm{x}_{\mathrm{k}}\right| \rho_{\mathrm{k}}
\end{aligned}
$$

Yukarıda tanımlanan $\|$.$\| fonksiyonu verilmiş olsun. \left(l_{\infty}(\mathrm{I}, \mathrm{K}, \rho),\|\cdot\|\right)$ çifti ultranormlu Banach uzayıdır. Benzer biçimde;

$$
\mathrm{c}_{0}(\mathrm{I}, \mathrm{K}, \rho)=\left\{\mathrm{x}=\left(\mathrm{x}_{\mathrm{k}}\right) \in \mathrm{K}: \lim _{\mathrm{k}}\left|\mathrm{x}_{\mathrm{k}}\right| \rho_{\mathrm{k}}=0\right\} \text { ve } \mathrm{c}(\mathrm{I}, \mathrm{K}, \rho)=\left\{\mathrm{x}=\left(\mathrm{x}_{\mathrm{k}}\right) \in \mathrm{K}: \lim _{\mathrm{k}}\left|\mathrm{x}_{\mathrm{k}}\right| \rho_{\mathrm{k}} \text { mevcut }\right\}
$$

kümeleri de $\|\mathrm{x}\|=\sup _{\mathrm{k}}\left|\mathrm{x}_{\mathrm{k}}\right| \rho_{\mathrm{k}}$ ile beraber Arşimedyan olmayan Banach uzaylarıdır $[1,8,9,10]$.

$l_{\infty}(\mathrm{I}, \mathrm{K}, \rho)$ ultra sınırlı dizilerin uzayı, $\mathrm{c}_{0}(\mathrm{I}, \mathrm{K}, \rho)$ ultra sıfıra yakınsak dizilerin uzayı, $\mathrm{c}(\mathrm{I}, \mathrm{K}, \rho)$ ultra yakınsak dizilerin uzayı olarak isimlendirilir.

Aşağıda yalnız $l_{\infty}(\mathrm{I}, \mathrm{K}, \rho)$ ultra sınırlı dizisinin ultra Banach uzayı olduğu gösterilecek olup diğerleri de benzer şekilde gösterilebilir.

i ) Ultranorm olduğu gösterilir.

$$
\begin{aligned}
& \text { (un1) }\|\mathrm{x}\|=\sup _{\mathrm{k}}\left|\mathrm{x}_{\mathrm{k}}\right| \rho_{\mathrm{k}}=0 \Leftrightarrow\left|\mathrm{x}_{\mathrm{k}}\right| \rho_{\mathrm{k}}=0 \Leftrightarrow\left|\mathrm{x}_{\mathrm{k}}\right|=0 \Leftrightarrow \mathrm{x}_{\mathrm{k}}=0 \Leftrightarrow \mathrm{x}=0 \text {, } \\
& \text { (un2) }\|\alpha x\|=\sup _{\mathrm{k}}\left|\alpha \mathrm{x}_{\mathrm{k}}\right| \rho_{\mathrm{k}}=|\alpha| \sup _{\mathrm{k}}\left|\mathrm{x}_{\mathrm{k}}\right| \rho_{\mathrm{k}}=|\alpha|\|\mathrm{x}\| \text { dir. } \\
& \text { (un3) }\|\mathrm{x}+\mathrm{y}\|=\sup _{\mathrm{k}}\left|\mathrm{x}_{\mathrm{k}}+\mathrm{y}_{\mathrm{k}}\right| \rho_{\mathrm{k}} \leq \sup _{\mathrm{k}}\left\{\operatorname{mak}\left\{\left|\mathrm{x}_{\mathrm{k}}\right| \rho_{\mathrm{k}},\left|\mathrm{y}_{\mathrm{k}}\right| \rho_{\mathrm{k}}\right\}\right. \\
& =\sup _{\mathrm{k}}\left\{\operatorname{mak}\left\{\left|\mathrm{x}_{\mathrm{k}}+\theta\right| \rho_{\mathrm{k}},\left|\mathrm{y}_{\mathrm{k}}+\theta\right| \rho_{\mathrm{k}}\right\}\right. \\
& \leq \sup _{\mathrm{k}}\left\{\operatorname{mak}\left\{\operatorname{mak}\left\{\left|\mathrm{x}_{\mathrm{k}}\right| \rho_{\mathrm{k}}, \theta\right\}, \operatorname{mak}\left\{\left|\mathrm{y}_{\mathrm{k}}\right| \rho_{\mathrm{k}}, \theta\right\}\right\}\right\} \\
& =\operatorname{mak}\left\{\sup _{\mathrm{k}}\left\{\operatorname{mak}\left\{\left|\mathrm{x}_{\mathrm{k}}\right| \rho_{\mathrm{k}}, \theta\right\}, \sup _{\mathrm{k}}\left\{\operatorname{mak}\left\{\left|\mathrm{y}_{\mathrm{k}}\right| \rho_{\mathrm{k}}, \theta\right\}\right\}\right\}\right. \\
& =\operatorname{mak}\left\{\sup _{\mathrm{k}} \mathrm{d}\left(\mathrm{x}_{\mathrm{k}}, \theta\right), \sup _{\mathrm{k}} \mathrm{d}\left(\mathrm{y}_{\mathrm{k}}, \theta\right)\right\}=\operatorname{mak}\{\|\mathrm{x}\|,\|\mathrm{y}\|\} .
\end{aligned}
$$

Dolayısıyla $l_{\infty}(\mathrm{I}, \mathrm{K}, \rho)$ ultranormlu uzaydır.

ii)Tam olduğu ispatlanır. 
Varsayalım ki $\left(\mathrm{x}^{\mathrm{n}}\right), l_{\infty}(\mathrm{I}, \mathrm{K}, \rho)$ de bir Cauchy dizisi olarak verilsin. $\left(\mathrm{x}^{\mathrm{n}}\right)$ in sabit bir dizi olması durumunda tamlık açıktır.

Diğer durumda $\left(x^{n}\right)$ in sabit bir dizi olmadığı düşünülürse bu takdirde $m, n \geq n_{0}$ olmak üzere

$$
\left\|x^{m}-x^{n}\right\|=\sup _{k \in I}\left|x_{k}^{m}-x_{k}{ }^{n}\right| \rho_{k}<\varepsilon
$$

için gerçeklenir.

Öyle ki $\varepsilon>0$ ve $\mathrm{k}=1,2, \ldots$ olacak şekilde keyfi lakin sabit her $\mathrm{k}$ için $\mathrm{m}, \mathrm{n} \geq \mathrm{n}_{0}$ iken $\left|\mathrm{x}_{\mathrm{k}}{ }^{\mathrm{m}}-\mathrm{x}_{\mathrm{k}}{ }^{\mathrm{n}}\right|<\varepsilon$ elde edilir ve bir $\mathrm{n}_{0}$ tam sayısının var olduğu gözlemlenir. Diğer taraftan her sabit $\mathrm{k}$ için $\left(\mathrm{x}_{\mathrm{k}}^{1}, \mathrm{x}_{\mathrm{k}}^{2}, \mathrm{x}_{\mathrm{k}}^{3}, \ldots\right) \mathrm{K}$ ' de bir Cauchy dizisidir. $K$ tam olduğu bilindiğine göre $\left(x_{k}^{m}\right) \rightarrow x_{k} \in K$ yazılabilir. $k$ doğal sayısının her bir değeri için elde edilecek limitler yardımıla $\mathrm{K}$ ' de $\mathrm{x}=\left(\mathrm{x}_{1}, \mathrm{x}_{2}, \ldots\right)$ dizisi teşkil edilir.

$$
\begin{aligned}
& x_{1}=\left(x_{1}^{1}, x_{2}^{1}, \ldots, x_{n}^{1}, \ldots\right) \\
& x_{2}=\left(x_{1}^{2}, x_{2}^{2}, \ldots, x_{n}^{2}, \ldots\right) \\
& x_{3}=\left(x_{1}^{3}, x_{2}^{3}, \ldots, x_{n}^{3}, \ldots\right) \\
& \vdots \\
& x_{k}=\left(x_{1}^{k}, x_{2}^{k}, \ldots, x_{n}^{k}, \ldots\right) \\
& \vdots \quad x_{m}=\left(x_{1}^{\mathrm{m}}, x_{2}^{\mathrm{m}}, \ldots, x_{n}^{m}, \ldots\right) \\
& \downarrow \quad \downarrow \quad \downarrow \quad \downarrow \quad \downarrow . \\
& x^{2}=\left(x_{1}, x_{2}, \ldots, x_{n}, \ldots\right)
\end{aligned}
$$

Burada ise $\mathrm{x} \in l_{\infty}(\mathrm{I}, \mathrm{K}, \rho)$ ve $\mathrm{n} \rightarrow \infty, \mathrm{x}_{\mathrm{n}} \rightarrow \mathrm{x}$ olduğu gösterilecektir. (*) ifadesinde $\mathrm{n} \rightarrow \infty$ yapılırsa $\left\|\mathrm{x}_{\mathrm{k}}{ }^{\mathrm{n}}-\mathrm{x}_{\mathrm{k}}\right\|_{l_{\infty}(\mathrm{I}, \mathrm{K}, \rho)}<\varepsilon$ elde edilir.

$\mathrm{x}_{\mathrm{n}}=\left(\mathrm{x}_{\mathrm{k}}^{\mathrm{n}}\right) \in l_{\infty}(\mathrm{I}, \mathrm{K}, \rho)$ varlığından $\mathrm{k}=1,2, \ldots$ için $\left\|\mathrm{x}_{\mathrm{k}}{ }^{\mathrm{n}}\right\| \leq \mathrm{t}_{\mathrm{n}}$ olmak üzere bir $\mathrm{t}_{\mathrm{n}}$ reel dizisi vardır.

Güçlü üçgen eşitsizliğinden;

$\left\|x_{k}\right\| \rho_{k}=\left\|x_{k}-x_{k}{ }^{n}+x_{k}{ }^{n}\right\| \rho_{k} \leq \operatorname{mak}\left\{\left\|x_{k}-x_{k}{ }^{n}\right\|,\left\|x_{k}{ }^{n}\right\|\right\} \rho_{k} \leq \operatorname{mak}\left\{\varepsilon, t_{n}\right\} M<\infty$.

Yukarıdaki eşitsizliğe bakıldığında her k sayısı için geçerlidir. Ayrıca eşitsizliğin sağ tarafı k’ yi içermediğinden $\left(x_{k}\right) \in l_{\infty}(I, K, \rho)$ dir. $l_{\infty}(I, K, \rho)$ ultra Banach uzayıdır.

$$
\begin{aligned}
& \text { Şimdi } Z: \tilde{l}_{\infty}(I, K, \rho) \rightarrow l_{\infty}(I, K, \rho) \\
& x \rightarrow Z x=y, \quad y=y_{k} \\
& y_{k}=p_{k}+(1-p) x_{k-1} \text { dönüşümü kullanılırsa görülür ki } \tilde{l}_{\infty}(I, K, \rho) \text { ile } l_{\infty}(I, K, \rho) \text { uzayları ultra }
\end{aligned}
$$
izometriktir ve ultra izomorfiktir.

i) Z nin lineer olduğu gösterilir.

$\forall \alpha \in \mathrm{K}$ ve $\forall \mathrm{x}, \mathrm{y} \in \tilde{\lambda}(\mathrm{I}, \mathrm{K}, \rho)$ için 


$$
\begin{aligned}
& Z(x+y)=p\left(x_{k}+y_{k}\right)+(1-p)\left(x_{k-1}+y_{k-1}\right) \\
& =p_{k}+p y_{k}+(1-p) x_{k-1}+(1-p) y_{k-1} \\
& =p x_{k}+(1-p) x_{k-1}+p y_{k}+(1-p) y_{k-1} \\
& =Z(x)+Z(y) \\
& Z(\alpha x)=p\left(\alpha x_{k}\right)+(1-p)\left(\alpha x_{k-1}\right) \\
& =\alpha\left(p x_{k}+(1-p) x_{k-1}\right)=\alpha Z(x) \text { dir. }
\end{aligned}
$$

ii) $Z$ nin örten olduğu gösterilir.

$\mathrm{Z}: \tilde{l}_{\infty}(\mathrm{I}, \mathrm{K}, \rho) \rightarrow l_{\infty}(\mathrm{I}, \mathrm{K}, \rho)$

$x \rightarrow z x=y, \quad y=y_{k}$

$\mathrm{x}=\mathrm{Z}^{-1} \mathrm{y}$ iken $\tilde{l}_{\infty}(\mathrm{I}, \mathrm{K}, \rho)$ da her $\mathrm{x}=\left(\mathrm{x}_{\mathrm{k}}\right)=\sum_{\mathrm{j}=0}^{\mathrm{k}}(-1)^{\mathrm{k}-\mathrm{j}} \frac{(1-\mathrm{p})^{\mathrm{k}-\mathrm{j}}}{\mathrm{p}^{\mathrm{k}-\mathrm{j}+1}} \mathrm{y}_{\mathrm{j}}$

$l_{\infty}(\mathrm{I}, \mathrm{K}, \rho)$ da yukarıdaki yazılan formda bir y elemanı bulunur ki, Z nin örtenliği ispatlanmış olur.

iii ) Z nin ultra izometri yani ultranormu koruduğu gösterilir.

$$
\begin{aligned}
& \mathrm{x}_{\mathrm{k}}=\sum_{\mathrm{j}=0}^{\mathrm{k}}(-1)^{\mathrm{k}-\mathrm{j}} \frac{(1-\mathrm{p})^{\mathrm{k}-\mathrm{j}}}{\mathrm{p}^{\mathrm{k}-\mathrm{j}+1}} \mathrm{y}_{\mathrm{j}} \text { dizisini tanımlayalım. } \\
\|\mathrm{x}\| & =\sup _{\mathrm{k}}\left|\mathrm{px}_{\mathrm{k}}+(1-\mathrm{p}) \mathrm{x}_{\mathrm{k}-1}\right| \rho_{\mathrm{k}} \\
& =\sup _{\mathrm{k}}\left|\mathrm{p} \sum_{\mathrm{j}=0}^{\mathrm{k}}(-1)^{\mathrm{k}-\mathrm{j}} \frac{(1-\mathrm{p})^{\mathrm{k}-\mathrm{j}}}{\mathrm{p}^{\mathrm{k}-\mathrm{j}+1}} \mathrm{y}_{\mathrm{j}}+(1-\mathrm{p}) \sum_{\mathrm{j}=0}^{\mathrm{k}}(-1)^{\mathrm{k}-\mathrm{j}} \frac{(1-\mathrm{p})^{\mathrm{k}-\mathrm{j}}}{\mathrm{p}^{\mathrm{k}-\mathrm{j}+1}} \mathrm{y}_{\mathrm{j}}\right| \rho_{\mathrm{k}} \\
& =\sup _{\mathrm{k}}\left|\mathrm{y}_{\mathrm{k}}\right| \rho_{\mathrm{k}}=\sup _{\mathrm{k}}|\mathrm{Zx}| \rho_{\mathrm{k}}=\|\mathrm{Zx}\|_{\lambda(\mathrm{I}, \mathrm{K}, \rho)} \operatorname{dir} .
\end{aligned}
$$

İzometrik dönüşümlerin 1:1 bir dönüşüm olduğu bilindiğinden dolayı bu durum ultra izometri içinde geçerliliği açıktır, tekrar gösterime gerek olmadığından $Z$ birebirdir.

Dolayısıyla $Z$ dönüşümü lineer, birebir, örten ve ultranormu koruyan ultra izometrik dönüşümdür. Özetle $\tilde{l}_{\infty}(\mathrm{I}, \mathrm{K}, \rho)$ ile $l_{\infty}(\mathrm{I}, \mathrm{K}, \rho)$ lineerdir ve ultra izometrik olarak ultra izomorfik uzaylardır. Şayet $\rho_{\mathrm{k}}=1$ alınması halinde $c_{0}(I, K, \rho)$ uzayı $c_{0}$ sıfıra yakınsak dizilerin uzayına, $c(I, K, \rho)$ de c yakınsak dizilerin uzayına son olarak da $l_{\infty}(\mathrm{I}, \mathrm{K}, \rho)$ bilinen anlamıyla $l_{\infty}$ sınırlı dizilerin uzayına dönüştükleri görülür.

\section{SONUÇLAR}

$\mathrm{Bu}$ çalışmada ultrametrik, ultranorm, ultra Banach uzayları incelenmiş, teoremler ve örnekler verilmiş̧ir. Sonuç olarak ultrametrik uzayların metrik uzaylar tarafından kapsanan daha spesifik yapılar olduğu gözlemlenmiş ve metrik uzaylarda var olan özelliklerin tamamının ultrametrik uzaylarda mevcut olmadığı belirlenmiştir. Ayrıca ultrametrikler daha özel oldukları için şaşırtan bazı sonuçlara ulaşılmıştır. Son bölümde ise ultra Banach uzayına örnekler verilmiş, ispatlanmış ve ultra izomorfik olduğu gösterilmiştir.

\section{KAYNAKLAR}

[1] Diagana, T. (2006). An Introduction to Classical and p-ADIC Theory of Linear Operators and Applications, by Nova Science Publishers Inc, ISBN 1-59454-424-7, New York. 
[2] Krull, W. (1998). Ultrametric Triangle Inequality. Planet Math, Göttingen 1959.Li, G., Hart, A. ve Gregory, J., Flocculation and sedimentation, 295, Technomics Press. Lancaster PA.

[3] Ludkovsky. S., and Diarra, B. (2002). Spectral Integration and Spectral Theory for Non-Arcimedean Banach Spaces IJMMS 31,7, 421-442.

[4] Şanlıbaba, I. (2014). Ultrametric Banach space isomorphic to new spaces, Master's thesis. Nevşehir Hacı Bektaş Veli University, Nevşehir.

[5] Bayraktar, M. (2006). Fonsiyonel Analiz. Gazi Kitabevi. Ankara.

[6] Nesin, A. (2012). Analiz IV. Nesin Matematik Köyü, İstanbul.

[7] Gajic, L. (2001). On Ultrametric Space, Novi Sad J. Match. 31, 2, 69-71.

[8] Diagana, T. (2006). $\mathrm{c}_{0}$ - Semigroups of Linear Operators on some Ultrametric Banach Space. IJMMS, DOI10. 1155/2006/52398.

[9] Diagana, T. (2007). Non-Arcimedean Linear Operators and Applications, by Nova Science Publishers Inc. ISBN 1-60021-405-3, New York.

[10] Diarra B. (1998). An Operator on Ultrametric Hilbert Spaces. Journal of Analysis 6, 55-74.

[11] Kaplansky, I. (1972). Set Theory and Metric Spaces. AMS Chelsea Publishing, ISBN 0-8218-2694-8.

[12] Perez-Garcia, C., Schikhof, W. H. (2010). Locally Convex Spaces over Non-Archimedean Valued Fields. Cambridge University Press, 978-0-521-19243-9.

[13] Havinga, M. (2011). Ultrametric Matrices, Korteweg-de Vries Institute for Mathematics Faculty of Science. $5-14,13$. 